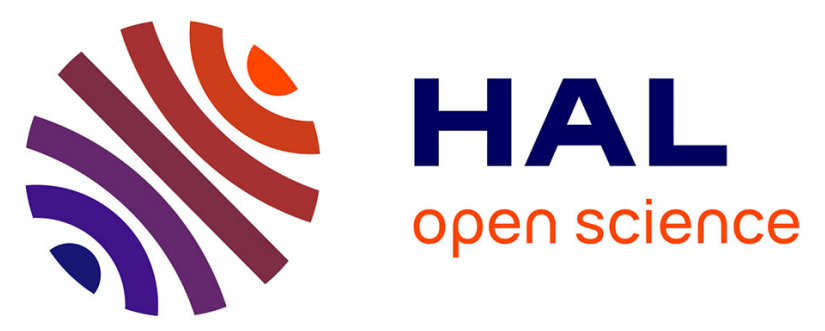

\title{
Urinary prostaglandin D2 and E2 metabolites associate with abdominal obesity, glucose metabolism, and triglycerides in obese subjects
}

Sven-Christian Pawelzik, Antoine Avignon, Helena Idborg, Catherine Boegner, Françoise Stanke-Labesque, Per-Johan Jakobsson, Ariane Sultan, Magnus Bäck

\section{To cite this version:}

Sven-Christian Pawelzik, Antoine Avignon, Helena Idborg, Catherine Boegner, Françoise StankeLabesque, et al.. Urinary prostaglandin D2 and E2 metabolites associate with abdominal obesity, glucose metabolism, and triglycerides in obese subjects. Prostaglandins and Other Lipid Mediators, 2019, 145, pp.106361. 10.1016/j.prostaglandins.2019.106361 . hal-02267011

\section{HAL Id: hal-02267011 \\ https://hal.science/hal-02267011}

Submitted on 17 Jun 2020

HAL is a multi-disciplinary open access archive for the deposit and dissemination of scientific research documents, whether they are published or not. The documents may come from teaching and research institutions in France or abroad, or from public or private research centers.
L'archive ouverte pluridisciplinaire $\mathbf{H A L}$, est destinée au dépôt et à la diffusion de documents scientifiques de niveau recherche, publiés ou non, émanant des établissements d'enseignement et de recherche français ou étrangers, des laboratoires publics ou privés. 


\title{
Urinary prostaglandin $\mathrm{D}_{2}$ and $\mathrm{E}_{2}$ metabolites associate with abdominal obesity, glucose metabolism, and triglycerides in obese subjects
}

\author{
Sven-Christian Pawelzik ${ }^{\mathrm{a}, \mathrm{b}, *}$, Antoine Avignon ${ }^{\mathrm{c}, \mathrm{d}}$, Helena Idborg ${ }^{\mathrm{e}}$, Catherine Boegner ${ }^{\mathrm{c}}$, \\ Françoise Stanke-Labesque ${ }^{\mathrm{f}}$, Per-Johan Jakobsson ${ }^{\mathrm{e}}$, Ariane Sultan ${ }^{\mathrm{c}, \mathrm{d}, 1}$, Magnus Bäck ${ }^{\mathrm{a}, \mathrm{b}, \mathrm{g}, \mathrm{h}, 1}$ \\ ${ }^{a}$ Cardiovascular Medicine Unit, Department of Medicine Solna, Karolinska Institutet, Stockholm, Sweden \\ b Theme Heart and Vessels, Division of Valvular and Coronary Disease, Karolinska University Hospital, Stockholm, Sweden \\ ${ }^{\mathrm{c}}$ Endocrinology-Diabetology-Nutrition Department, CHRU Montpellier, Montpellier, France \\ ${ }^{\mathrm{d}}$ INSERM U1046, Université Montpellier 1, Montpellier, France \\ ${ }^{\text {e }}$ Rheumatology Unit, Department of Medicine Solna, Karolinska Institutet, Stockholm, Sweden \\ ${ }^{\mathrm{f}}$ INSERM U1042, Université de Grenoble, Grenoble, France \\ ${ }^{\mathrm{g}}$ INSERM U1116, Université de Lorraine, Nancy, France \\ ${ }^{\mathrm{h}}$ CHRU Nancy, Vandoeuvre-Lès-Nancy, France
}

Keywords:

Obesity

Cyclooxygenase (COX)

Inflammation

Tetranor-PGEM

Tetranor-PGDM

Metabolic syndrome

\begin{abstract}
A B S T R A C T
Obesity is associated with low-grade chronic inflammation, which contributes to the development of the metabolic syndrome and its associated complications, such as insulin resistance and type-2 diabetes. Limited data from animal and human studies support local generation of pro-inflammatory prostanoid lipid mediators in white adipose tissue. However, the link between systemic prostanoid levels and parameters characterizing the metabolic syndrome is missing in human obesity. Therefore, we performed a targeted lipidomic analysis using urine samples from obese human subjects $(n=45)$ and show for the first time in humans that urinary prostanoid levels correlate with metabolic parameters that indicate a dysregulated glucose and triglyceride metabolism. We identified tetranor-PGDM and tetranor-PGEM as the two major urinary prostanoid metabolites in obese subjects with levels of $247 \pm 31$ and $23.3 \pm 4.0 \mathrm{pmol} / \mathrm{mg}$ creatinine, respectively. Tetranor-PGDM was significantly associated with serum triglycerides, while tetranor-PGEM was associated with abdominal obesity as defined by an increased waist-to-hip ratio (WHR), with glycated hemoglobin (HbA1c), and with impaired oral glucose tolerance. These results confirm the previously established notion of low-grade chronic inflammation in obesity and further identify an association of the prostanoid pathway with obesity-associated dyslipidemia, abdominal obesity, and insulin resistance.
\end{abstract}

\section{Introduction}

During the past decades, the globally advancing epidemic of obesity, defined as the excessive accumulation of white adipose tissue (WAT) with a body mass index (BMI) exceeding $30 \mathrm{~kg} / \mathrm{m}^{2}$, has been recognized by the World Health Organization (WHO) as a neglected public health problem. Besides diminishing quality of life and overall life expectancy, obesity contributes as a risk factor to several associated diseases, particularly cardiovascular diseases (CVD) and type 2 diabetes. Obesity is considered the sixth most important risk factor for the overall burden of disease worldwide [1], and its management is key to the prevention of cardiovascular events $[2,3]$.

Recent studies have identified signs of obesity-induced chronic inflammation, which is reflected in higher numbers of activated macrophages in the WAT and elevated concentrations of circulating systemic markers of inflammation in obese subjects [4]. An activation of arachidonic acid metabolism has also been established in obesity, leading to the formation of pro-inflammatory lipid mediators. Obesity is for instance an independent predictor of urinary leukotriene $\mathrm{E}_{4}\left(\mathrm{LTE}_{4}\right)$ in subjects with obstructive sleep apnea [5]. Furthermore, we have previously shown that urinary $\mathrm{LTE}_{4}$ correlates with abdominal obesity in the present cohort [6]. The picture is, however, more complex for prostanoid lipid mediators, which are also formed from arachidonic acid. Prostanoids are generated by the concerted action of cyclooxygenase (COX) plus a specific, terminal prostanoid synthase leading to the primary prostanoids, prostaglandin $\mathrm{D}_{2}\left(\mathrm{PGD}_{2}\right), \mathrm{PGE}_{2}, \mathrm{PGF}_{2 \alpha}, \mathrm{PGI}_{2}$

* Corresponding author at: Cardiovascular Medicine Unit, Department of Medicine Solna, Karolinska Institutet, Stockholm, Sweden.

E-mail address: Sven-Christian.Pawelzik@ki.se (S.-C. Pawelzik).

${ }^{1}$ Equal contribution. 
(prostacyclin), and thromboxane (TX)A $\mathrm{A}_{2}$. COX-2, the central enzyme in the pathway, is upregulated in obesity [7] as well as in CVD [8]. $\mathrm{PGE}_{2}$ is usually low to undetectable at basal levels, but highly inducible under inflammatory conditions [9]. In murine WAT of lean animals, however, microsomal $\mathrm{PGE}_{2}$ synthase 1 (MPGES1) is abundantly expressed at considerably higher basal levels than in all other tissues [10]. Interestingly, its expression was consistently 3- to 4-fold higher in lean epididymal WAT (eWAT, corresponding to visceral WAT in humans) compared to lean inguinal WAT (iWAT, corresponding to cutaneous WAT in humans), indicating a depot-dependent regulation and importance of $\mathrm{PGE}_{2}$ biosynthesis in eWAT [10]. Surprisingly, this study showed a significant downregulation of MPGES1 in both eWAT and iWAT in high-fat diet-induced obesity, which was reflected on the metabolite level [10]. However, other studies found tissue levels of $\mathrm{PGE}_{2}$ in the eWAT of rats to be significantly increased with diet-induced obesity [11]. Physical activity reduced the eWAT levels of $\mathrm{PGE}_{2}$ significantly along with fractional visceral adiposity of these animals. A human study comparing visceral WAT from obese and non-obese subjects, respectively, detected no differences in mRNA and protein levels for any of the three $\mathrm{PGE}_{2}$ synthase isozymes, however, expression of COX-2 was significantly increased in visceral WAT of obese subjects [7]. This was consistent with significantly higher tissue levels of $\mathrm{PGE}_{2}$ in the visceral WAT of obese humans. Furthermore, there was a positive correlation between COX-2 mRNA expression and increasing BMI, whereas MPGES1 mRNA expression showed no such correlation. Increased production of $\mathrm{PGE}_{2}$ in WAT of obese humans therefore seems to be driven by COX-2.

Diet-induced obese mice showed also perturbed levels of $\mathrm{PGD}_{2}$ signaling in WAT, which was persistent even after weight loss [12] and predominantly produced by WAT macrophages involving the hematopoietic isoform of prostaglandin $\mathrm{D}_{2}$ synthase (H-PGDS) [13]. H-PGDS expression was about 10-fold upregulated in WAT of high-fat diet fed mice compared to lean littermates, while lipocaline-type PGDS (LPGDS) expression remained unchanged in these animals [12]. In human WAT biopsies taken from lean or obese patients, the same study detected an approximately 2-fold increased expression of H-PGDS in obesity. Increased H-PGDS expression was consistent with a 10-fold increase in local tissue $\mathrm{PGD}_{2}$ in WAT of obese mice as well as with about 2-fold higher levels of circulating plasma $\mathrm{PGD}_{2}$ in obese human subjects compared to the clinical reference range for plasma $\mathrm{PGD}_{2}$ in normal individuals [12]. Mice deficient in L-PGDS, on the other hand, become glucose-intolerant and insulin-resistant at an accelerated rate, have increased fat depots, and show adipocytes with a significantly larger phenotype, even when they are not challenged by high-fat diet. L-PGDS knock-out mice also develop diet-induced atherosclerosis and diabetic nephropathy [14].

Prostanoids are ubiquitously formed throughout the body but act as autocrine or paracrine mediators only in a limited range in the direct vicinity of their site of generation. They are rapidly degraded to a series of biologically inactive metabolites that can be detected in the plasma and urine [15-17]. To be used as clinical markers reflecting disease state, urinary prostanoid metabolites are generally considered to be more reliable than their plasma-borne precursors [18-20] because they integrate systemic prostanoid formation over time. Furthermore, metabolic conversion is largely completed for the urinary metabolites, resulting in more stable levels that are not affected by ongoing metabolism. Measuring the final, urinary metabolites also excludes prostanoids from the analysis that are locally generated in the kidney and might otherwise tamper with the levels of systemic prostanoid formation. Tetranor-PGDM, the major urinary metabolite of $\mathrm{PGD}_{2}$, has been suggested as a biomarker in systemic mastocytosis and rheumatoid arthritis [21], to assess Aspirin-intolerant asthma [22], and allergic reactions of food allergy [23], while tetranor-PGEM, the major urinary metabolite of $\mathrm{PGE}_{2}$, has been suggested as biomarker for ulcerative colitis [24] and inflammation in infants [18].

In the present study, we aimed to determine the association of different prostanoids with metabolic and anthropometric factors in obesity. To this end, we used a targeted lipidomics analysis for urinary prostanoid metabolites in a cohort of well phenotyped obese subjects.

\section{Materials and methods}

\subsection{Study population}

Patients that were admitted to the University Hospital of Montpellier (France) for assessment of obesity (BMI $\geq 30 \mathrm{~kg} / \mathrm{m}^{2}$ ) were included as subjects in this study between January 2011 and February 2012. Exclusion criteria were treatment with anti-inflammatory medications, asthma, known diabetes mellitus (hemoglobin A1c (HbA1c) > 6,5\%; fasting plasma glucose $>7 \mathrm{mmol} / \mathrm{l}$ and/or treatment with any anti-diabetic medications), and hs-CRP above $10 \mathrm{mg} / 1$. All participants had normal cardiopulmonary functions as determined by medical history, physical examination and electrocardiogram. Height and weight were measured wearing light clothing and no shoes. BMI was calculated as weight in $\mathrm{kg}$ divided by the square of height in meters $\left(\mathrm{kg} / \mathrm{m}^{2}\right)$. Waist circumference was recorded to the nearest $0.1 \mathrm{~cm}$ midway between the last rib and the iliac crest using a non-stretch tape measure on lightly clad participants.

All clinical investigations were conducted according to the principles expressed in the Declaration of Helsinki and in compliance with International Conference on Harmonization/Good Clinical Practice regulations. After approval by the Montpellier University Hospital's ethics committee (Comité de Protection des Personnes Sud Méditerranée IV), the study was registered at the Ministère de la Santé et de l'Enseignement Supérieur according to French law; study number DC-2009-1052. All patients gave their written informed consent. For minor participants, written informed consent were obtained from the legal guardian.

\subsection{Serum collection and measurements}

Blood was drawn in the morning after an $8 \mathrm{~h}$ overnight fasting period. Plasma glucose and glycated hemoglobin (HbA1c) were analyzed by routine high-performance liquid chromatography (HPLC)based ion-exchange procedure (HA-8140; Menarini, Rungis Cedex, France). Total cholesterol, triglycerides (TG), high density lipoprotein (HDL), and creatinine were measured using routine enzymatic methods (KonePro; Konelab, Epoo, Finland). LDL cholesterol was calculated according to the Friedwald formula. Hs-CRP was assessed by immunoturbidimetry (Randox reagents on Olympus apparatus, Rungis, France). The Homeostatic Model Assessment Index-insulin Resistance (HOMA-IR) was calculated as follows: fasting insulin $\times$ fasting glucose/ 22.5 (insulin-resistance was defined by a HOMA-IR $\geq 3$ ) [25].

\subsection{Urine collection and measurements}

Urine was collected in the morning and frozen in aliquots at $-80{ }^{\circ} \mathrm{C}$ until analysis. Urinary prostanoid metabolites were measured in June 2012 by liquid chromatography-tandem mass spectrometry (LC-MS/ MS) as described before [18]. $600 \mu \mathrm{l}$ of urine were acidified and spiked with deuterated internal standard. External standard curves ranging from 0.05 to $18 \mathrm{pmol} / \mu \mathrm{l}$ were prepared accordingly using eicosanoid standards and MilliQ water as a surrogate matrix. All standards were purchased from Cayman Chemical, Ann Arbor, MI, USA. Samples were extracted on $30 \mathrm{mg}$ Oasis HLB Extraction 96 well Plates or single extraction columns (Waters Corporation, Milford, MA, USA), washed with $5 \%$ methanol in MilliQ water acidified with $0.05 \%$ formic acid (Merck $\mathrm{AB}$, Solna, Sweden), and retained material was eluted with methanol, which was collected and subsequently evaporated under vacuum. The dried samples were reconstituted in $7 \%$ acetonitrile in MilliQ water.

LC-MS/MS analysis was performed on a Waters 2795 HPLC coupled to an Acquity TQ Detector triple quadrupole mass spectrometer (Waters 
Corporation, Milford, MA, USA). Analytes were separated on a Synergi Hydro-RP column $(100 \mathrm{~mm}, 2 \mathrm{~mm}$ i.d., $2.5 \mu \mathrm{m}$ particle size and $100 \AA$ pore size, Phenomenex, Torrance, CA, USA) equipped with an appropriate precolumn using a mobile phase that composed of acidified MilliQ water (0.05\% formic acid) as solvent A and acidified acetonitrile (HPLC grade, Rathburn Chemicals Ltd., Scandinavian Genetec AB, Sweden, $0.05 \%$ formic acid) as solvent B. Baseline separation of the analytes was achieved by a $30 \mathrm{~min}$ linear stepwise gradient at a flow rate of $0.2 \mathrm{ml} / \mathrm{min}$.

Separated residues were detected by negative mode electrospray ionization using multiple reaction monitoring. Tetranor-PGEM $(9,15-$ dioxo-11 $\alpha$-hydroxy-13,14-dihydro-2,3,4,5-tetranor-prostan-1,20-dioic acid) and tetranor-PGDM (9 $\alpha$-hydroxy-11,15-dioxo-13,14-dihydro2,3,4,5-tetranor-prostan-1,20-dioic acid) were recorded at identical mass transitions $(m / z 327.2>309.2)$, and the respective deuterated internal standards were recorded at $\mathrm{m} / \mathrm{z} 333.2>315.2$. The lower limits of quantification (LLOQ) were $11.7 \mathrm{pmol}$ for tetranor-PGEM, and $23.4 \mathrm{pmol}$ for tetranor-PGDM. 2,3-dinor $\mathrm{TXB}_{2}(9 \alpha, 11,15 \mathrm{~S}$-trihydroxy2,3-dinor-thromba-5Z,13E-dien-1-oic acid) and 2,3-dinor 6-keto $\mathrm{PGF}_{1 \alpha}$ (6-oxo-9 $\alpha, 11 \alpha$, 15S-trihydroxy-2,3-dinor-prost-13E-en-1-oic acid) were recorded at mass transitions $m / z=341.4>123.1$ and $m /$ $z=341.4>135.1$, respectively, but no external standards for these compounds were used in the analysis. Instrument operation and data analysis was carried out using MassLynx software, version 4.1.

The obtained levels of tetranor-PGDM and tetranor-PGEM were normalized to urinary creatinine concentrations, which were determined using a standard colorimetric assay (Cayman Chemicals, Ann Arbor, MI, USA).

\subsection{Statistical analysis}

Data are expressed as average \pm SEM and frequencies are expressed as percentages. A two-tailed Student's $t$-test was used for comparisons between 2 groups. Correlations between the urinary biomarker concentrations and the clinical parameters were established by Pearson correlation. A multiple stepwise linear regression was performed to evaluate urinary biomarkers as predictors of the clinical parameters monitored. $\mathrm{p}<0.05$ was considered significant. Analyses were performed using SigmaPlot version 12 (Systat Software Inc).

\section{Results}

\subsection{Study cohort}

After the initial screen, 59 subjects were included in the study. Of these, 11 subjects were excluded because their CRP-levels exceeded $10 \mathrm{mg} / \mathrm{L}$ (defined as exclusion criteria in [6]). Another two subjects were excluded because of non-usable urine samples, resulting in a study cohort of 46 subjects. As previously reported, $70 \%$ of the cohort was of female sex [6]. For the present study, another subject was excluded because its levels for both tetranor-PGDM and tetranor-PGEM were below the detection limit of our mass spectrometry-based method. The clinical and biochemical characteristics of the study cohort of the remaining 45 subjects are summarized in Table 1.

\subsection{Substantial concentration differences and lack of correlations between urinary $P G D_{2}$ and $P G E_{2}$ metabolites}

Of all prostanoid metabolites that we monitored, tetranor-PGDM and tetranor-PGEM were the only two detected above the quantification limit of the method. Tetranor-PGDM and tetranor-PGEM are, like their precursors $\mathrm{PGD}_{2}$ and $\mathrm{PGE}_{2}$, stereoisomers with an identical molecular mass and ion transition pattern during mass spectrometric analysis. Therefore, these analytes were carefully separated by liquid chromatography to assure veritable identification and quantification (Fig. 1A).

After normalization to creatinine, the urinary levels of tetranor-
PGDM (247 $\pm 31 \mathrm{pmol} / \mathrm{mg}$ creatinine) were significantly higher than those of tetranor-PGEM $(23.3 \pm 4.0 \mathrm{pmol} / \mathrm{mg}$ creatinine; Table 1 and Fig. 1B) with a ratio (tetranor-PGDM/tetranor-PGEM) of $10.6 \pm 3.2$ $(n=42)$. There was no significant correlation between the two urinary analytes (Fig. 1C).

\subsection{Tetranor-PGEM, but not tetranor-PGDM, is associated with waist-to- hip-ratio in obese subjects}

All patients fulfilled the study inclusion criteria of obesity as assessed by BMI. The waist-to-hip-ratio (WHR) is used as an indicator to further characterize the obese phenotype, with a WHR $\geq 0.90$ for males and $\geq 0.85$ for females defined as abdominal obesity [26,27]. Stratification of our study population according to WHR revealed a fraction of $58 \%$ within the cohort of obese patients that also fulfilled the criteria for abdominal obesity.

Correlation analysis using these stratified subgroups revealed that the creatinine-normalized levels of tetranor-PGEM were significantly higher in subjects with an increased WHR ( $p=0.049$; Fig. 2A). Levels of tetranor-PGDM, on the other hand, did not significantly differ between the two subgroups (Fig. 2B).

\subsection{Metabolic parameters according to urinary prostanoid levels}

Since abdominal obesity is part of the metabolic syndrome, we next evaluated the relation between the urinary prostanoid metabolites and a range of metabolic parameters after stratification based on high and low urinary prostanoid levels. Subjects with normalized levels of tetranor-PGDM above median exhibited significantly higher concentrations of serum triglycerides compared with those below median, while total plasma cholesterol, HDL, or LDL showed no significant differences between these two groups (Table 1). Subjects with normalized levels of tetranor-PGEM above median exhibited significantly higher concentrations of HbA1c. Surprisingly, this group difference did not appear when HOMA-IR was assessed as additional measure of insulin resistance (Table 1). The significant associations between tetranor-PGDM with serum triglycerides and tetranor-PGEM with HbA1c are shown in Fig. 3A and Fig. 3B, respectively.

\subsection{Tetranor-PGEM and glucose tolerance test}

Further analysis of plasma glucose levels in relation to tetranorPGEM revealed a significant correlation with the 60-min plasma glucose of the oral glucose tolerance test (OGTT), whereas no significant associations were observed for either fasting plasma glucose or any of the other time points examined following glucose challenge (Table 2).

\section{Discussion}

Two major findings emerge from the present study. Firstly, using a targeted lipidomic approach, we identified tetranor-PGDM and tetranor-PGEM as abundant urinary prostanoid metabolites in our cohort of obese subjects, with levels of tetranor-PGDM that were more than 10fold higher than those of tetranor-PGEM. Secondly, metabolic parameters were associated with the prostanoid metabolite levels, with tetranor-PGEM being significantly linked to abdominal obesity, glycated hemoglobin (HbA1c) and impaired glucose tolerance, whereas tetranor-PGDM levels were significantly associated with serum triglycerides. Taken together, these findings indicate an upregulated $\mathrm{PGE}_{2}$ and $\mathrm{PGD}_{2}$ biosynthesis in obesity in humans with possible implications for metabolic control.

Interest in measuring urinary prostanoids in humans has emerged only recently when these metabolites have been identified as potential biomarkers for several diseases. In the present study, we measured both tetranor-PGEM and tetranor-PGDM in a cohort of obese subjects. One shortcoming of this study is the lack of a control group of non-obese 
Table 1

Patient characteristics.

\begin{tabular}{|c|c|c|c|c|c|c|c|}
\hline & All & high [tPGDM] & low [tPGDM] & $p$ & high [tPGEM] & low [tPGEM] & $p$ \\
\hline $\begin{array}{l}\text { [tPGEM] } \\
\qquad(\mathrm{pmol} / \mathrm{mg} \text { creatinine })\end{array}$ & $23.3 \pm 4.1$ & $27.7 \pm 8.0$ & $19.1 \pm 2.1$ & 0.295 & $35.6 \pm 7.4$ & $11.6 \pm 0.75$ & 0.002 \\
\hline $\begin{array}{l}\text { [tPGDM] } \\
\quad(\mathrm{pmol} / \mathrm{mg} \text { creatinine })\end{array}$ & $247 \pm 31$ & $396 \pm 40$ & $91.7 \pm 7.7$ & 4.2E-09 & $275 \pm 49$ & $218 \pm 37$ & 0.362 \\
\hline Age & $44 \pm 1.8$ & $43 \pm 2.6$ & $45 \pm 2.5$ & 0.726 & $45 \pm 3.0$ & $43 \pm 2.0$ & 0.704 \\
\hline Sex ( $\%$ female $)$ & 70 & 70 & 68 & NS & 61 & 77 & NS \\
\hline \multicolumn{8}{|l|}{ Smoking } \\
\hline current $(\%)$ & 13 & 22 & 5 & NS & 13 & 14 & NS \\
\hline former $(\%)$ & 5 & 9 & 14 & NS & 9 & 14 & NS \\
\hline BMI $\left(\mathrm{kg} / \mathrm{m}^{2}\right)$ & $38 \pm 0.87$ & $38 \pm 1.1$ & $39 \pm 1.3$ & 0.558 & $38 \pm 1.1$ & $38 \pm 1.4$ & 0.926 \\
\hline Waist $(\mathrm{cm})$ & $113 \pm 2.4$ & $111 \pm 2.9$ & $117 \pm 3.9$ & 0.209 & $115 \pm 3.3$ & $113 \pm 3.6$ & 0.645 \\
\hline W/H Ratio & $0.91 \pm 0.01$ & $0.9 \pm 0.02$ & $0.9 \pm 0.02$ & 0.344 & $0.9 \pm 0.02$ & $0.9 \pm 0.02$ & 0.710 \\
\hline Systolic BP (mmHg) & $123 \pm 2.0$ & $122 \pm 3.1$ & $124 \pm 2.5$ & 0.768 & $122 \pm 2.7$ & $124 \pm 3.0$ & 0.623 \\
\hline Diastolic BP (mmHg) & $73.4 \pm 1.1$ & $74 \pm 1.5$ & $73 \pm 1.9$ & 0.933 & $73 \pm 1.7$ & $74 \pm 1.7$ & 0.804 \\
\hline CRP & $4.3 \pm 0.43$ & $3.6 \pm 0.51$ & $5.0 \pm 0.68$ & 0.101 & $4.5 \pm 0.71$ & $4.1 \pm 0.49$ & 0.583 \\
\hline Chol $(g / 1)$ & $2.0 \pm 0.06$ & $2.0 \pm 0.07$ & $2.0 \pm 1.10$ & 0.946 & $1.9 \pm 0.09$ & $2.0 \pm 0.07$ & 0.382 \\
\hline LDL $(g / 1)$ & $1.2 \pm 0.05$ & $1.2 \pm 0.07$ & $1.3 \pm 0.07$ & 0.209 & $1.2 \pm 0.07$ & $1.3 \pm 0.06$ & 0.310 \\
\hline HDL $(g / 1)$ & $0.46 \pm 0.01$ & $0.5 \pm 0.02$ & $0.5 \pm 0.02$ & 0.897 & $0.5 \pm 0.02$ & $0.5 \pm 0.02$ & 0.764 \\
\hline $\mathrm{TG}(\mathrm{g} / 1)$ & $1.54 \pm 0.09$ & $1.7 \pm 0.15$ & $1.3 \pm 0.08$ & 0.032 & $1.6 \pm 0.13$ & $1.5 \pm 0.12$ & 0.552 \\
\hline \multicolumn{8}{|l|}{ Plasma glucose } \\
\hline Fasting & $5.1 \pm 0.1$ & $4.9 \pm 0.11$ & $5.2 \pm 0.16$ & 0.148 & $5.2 \pm 0.14$ & $5.0 \pm 0.14$ & 0.258 \\
\hline OGTT T30 & $8.1 \pm 0.28$ & $8.3 \pm 0.39$ & $7.9 \pm 0.40$ & 0.387 & $8.3 \pm 0.45$ & $7.8 \pm 0.27$ & 0.342 \\
\hline OGTT T60 & $8,9 \pm 0.40$ & $9.5 \pm 0.55$ & $8.4 \pm 0.56$ & 0.169 & $9.4 \pm 0.62$ & $8.4 \pm 0.46$ & 0.243 \\
\hline OGTT T90 & $7.8 \pm 0.34$ & $8.1 \pm 0.41$ & $7.6 \pm 0.55$ & 0.460 & $8.0 \pm 0.47$ & $7.6 \pm 0.49$ & 0.583 \\
\hline OGTT T120 & $7.1 \pm 0.25$ & $7.1 \pm 0.35$ & $7.1 \pm 0.35$ & 0.918 & $7.1 \pm 0.29$ & 7.20 .42 & 0.836 \\
\hline HOMA-IR & $3.4 \pm 0.34$ & $3.1 \pm 0.28$ & $3.7 \pm 0.64$ & 0.383 & $3.5 \pm 0.44$ & $3.4 \pm 0.54$ & 0.896 \\
\hline HbA1c & $5.8 \pm 0.08$ & $5.8 \pm 0.10$ & $5.9 \pm 0.12$ & 0.503 & $6.0 \pm 0.11$ & $5.7 \pm 0.10$ & 0.029 \\
\hline
\end{tabular}

All values represent the mean \pm SEM. tPGEM, tetranor-prostaglandin $\mathrm{E}_{2}$ metabolite; tPGDM, tetranor-prostaglandin $\mathrm{D}_{2}$ metabolite; BMI, body mass index; Chol, total cholesterol; OGTT, oral glucose tolerance test; $\mathrm{T}$, time (min).

individuals whose levels of urinary prostanoid metabolites could serve as a reference. Nonetheless, values of creatinine-normalized levels in the general population published in the literature could fill in this gap. Tetranor-PGEM was previously reported at levels in the low $\mathrm{ng} / \mathrm{mg}$ creatinine range in healthy, non-obese volunteers and found to be significantly increased after exercise, in COPD patients, asthma patients, and in infants with fever (Table 3). The levels that we measured in this study in obese adult subjects were at the higher end of the previously reported range (Table 1). These findings hint toward elevated levels of tetranor-PGEM with increasing BMI; especially in patients with abdominal obesity. Because increased tissue $\mathrm{PGE}_{2}$ is reflected on the systemic level as elevated concentration of tetranor-PGEM in the urine, the data that we report here are coherent with previous studies in rat [11] and humans [7] and support the notion of visceral WAT as a dominant source of systemic pro-inflammatory $\mathrm{PGE}_{2}$ levels, likely mediated by COX-2 regulation. In addition, we show that high levels of urinary tetranor-PGEM are associated with an increase in HbA1c, suggesting that $\mathrm{PGE}_{2}$ formation in visceral WAT may be linked to diabetes/ pre-diabetes in obese subjects. Indeed, further analysis revealed a significant correlation between tetranor-PGEM and increased glucose levels after OGTT, expanding the observation to indicate that $\mathrm{PGE}_{2}$ in obesity reflects insulin resistance. Previous studies of $\mathrm{PGE}_{2}$ in diabetes and glycemic control have shown that COX-2 mRNA expression in the eWAT of high-fat diet induced obese rats was highly correlated with HOMA-IR and plasma leptin levels. Treatment of rats with COX-2 specific inhibitors significantly suppressed the time-dependent elevation in tetranor-PGEM and reversed the elevated levels of plasma insulin, leptin and HOMA-IR [28]. While this suggests that $\mathrm{PGE}_{2}$ biosynthesis in visceral WAT inflammation is induced by COX-2 activation and important in the development of insulin resistance, we could not detect a correlation of tetranor-PGEM with HOMA-IR scores in the present study. A possible explanation for this is the relatively small sample size of our cohort.

Levels of tetranor-PGDM were also reported in the low $\mathrm{ng} / \mathrm{mg}$ creatinine range in healthy, non-obese volunteers. Tetranor-PGDM was found to be upregulated under various disease conditions, but decreased in patients with Aspirin tolerant asthma after challenge (Table 4). We measured here considerably higher levels of tetranorPGDM in obese subjects; on average a value that was 20 -fold higher than the reference values previously measured in healthy control subjects. This marked elevation in urinary tetranor-PGDM indicates an important role for $\mathrm{PGD}_{2}$ biosynthesis in obesity. Previous studies have measured the tissue levels of $\mathrm{PGD}_{2}$ in obese mice and circulating $\mathrm{PGD}_{2}$ in human plasma, respectively [12]. Like all primary prostanoids, $\mathbf{P G D}_{2}$ has a short half-life and is rapidly degraded to biologically inactive metabolites. Therefore, the tissue and plasma levels of $\mathrm{PGD}_{2}$ reported previously likely underestimate the real extent of $\mathrm{PGD}_{2}$ biosynthesis in obesity. Our measures of an about 20 -fold increase in urinary tetranorPGDM are in agreement with the previously described marked elevation of $\mathrm{PGD}_{2}$ in obesity but may represent a more accurate estimation of this biomarker for metabolic control. The present study also identified a weak, but significant correlation of tetranor-PGDM with serum triglyceride levels, which may be explained with an orexigenic role for $\mathrm{PGD}_{2}$ that perpetuates obesity in a positive feedback loop. Evidence for the importance of $\mathrm{PGD}_{2}$ in the control of food intake and regulation of body weight comes from animal studies that investigated signaling mechanisms in both the central nervous system [29] and the peripheral tissue in mice $[30,12]$. Intracerebroventricular injection of $\mathrm{PGD}_{2}$ was shown to stimulate food intake and promote body weight gain and fat mass increase in mice. Similar results were obtained with a specific agonist for the $\mathrm{PGD}_{2}$ receptor DP1, BW245C, but not with the DP2 receptor agonist 13,14-dihydro-15-keto- $\mathrm{PGD}_{2}$, while central administration of the DP1 antagonist BWA868C or antisense oligodeoxynucleotides directed against DP1 remarkably decreased food intake and associated adiposity [29]. These observations were expanded by the finding that peripheral administration of BW245C, but not 13,14dihydro-15-keto- $\mathrm{PGD}_{2}$, via intraperitoneal injection lowers food consumption and inhibits weight gain in mice [30]. Furthermore, peripheral treatment of these animals with BW245C slightly elevated the levels of leptin and lowered plasma glucose after OGTT. Finally, specific inhibition of H-PGDS has been tested as potential pharmacotherapy to support weight loss or prevent weight regain after weight loss. Oral 
A
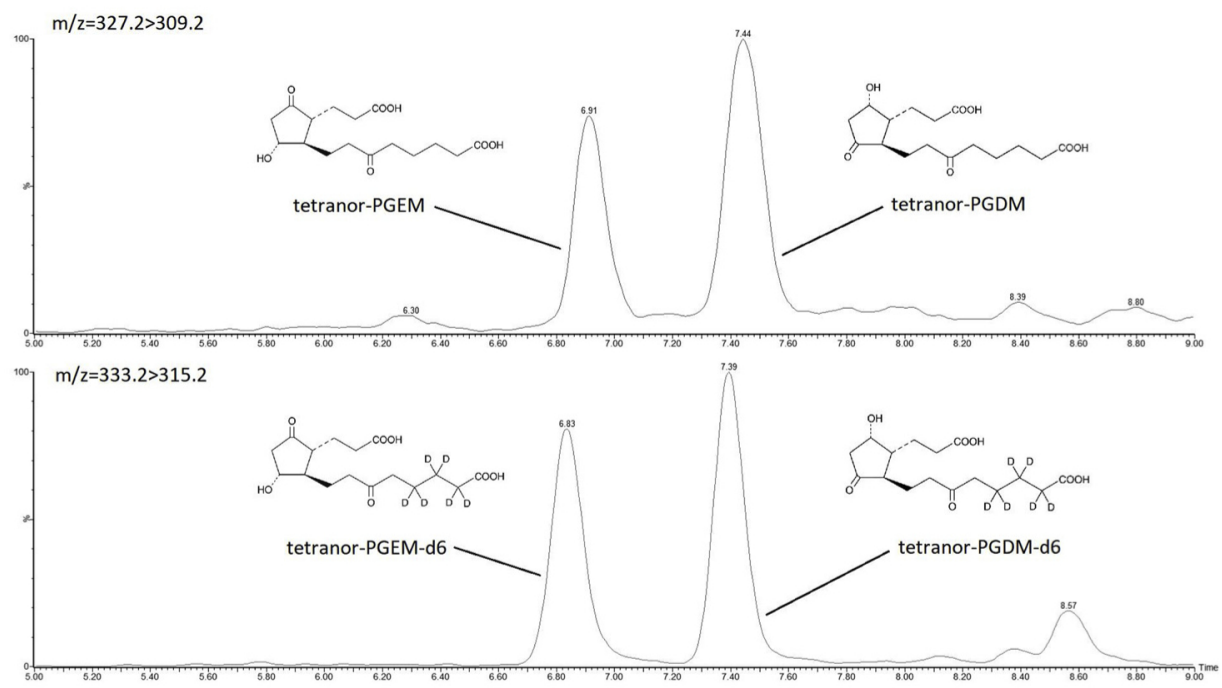

Fig. 1. Urinary prostanoid levels in obese human subjects. (A) Representative LC-MS/MS chromatogram of a urine sample. Liquid chromatography conditions were optimized to achieve baseline separation of the stereoisomers tetranor-PGEM and tetranor PGDM, which were subsequently detected in multiple reaction mode at the transition $m / z=327.2>309.2$ (upper panel). Deuterated analogues of tetranor-PGEM and tetranor PGDM were added as internal standard to each urine sample and simultaneously detected at the transition $m / z=333.2>315.2$. (B) Creatinine-normalized levels of tetranor-PGDM and tetranor-PGEM in urine from obese human subjects. The box plots show the median (horizontal line), interquartile range (box), and range (whiskers). Outliers are depicted as individual data points. $(\mathrm{n}=45, \mathrm{p}<0.0001)$. (C) Correlation analysis failed to detect a significant correlation between creatinine-normalized levels of tetranor-PGDM and tetranor-PGEM in obese human subjects. ( $r=0.203, \mathrm{p}=0.17$ ).

B

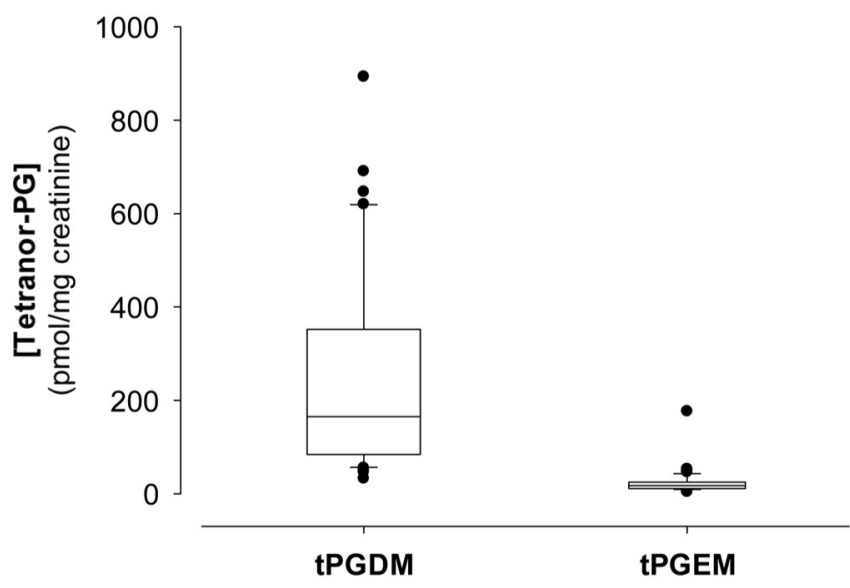

C

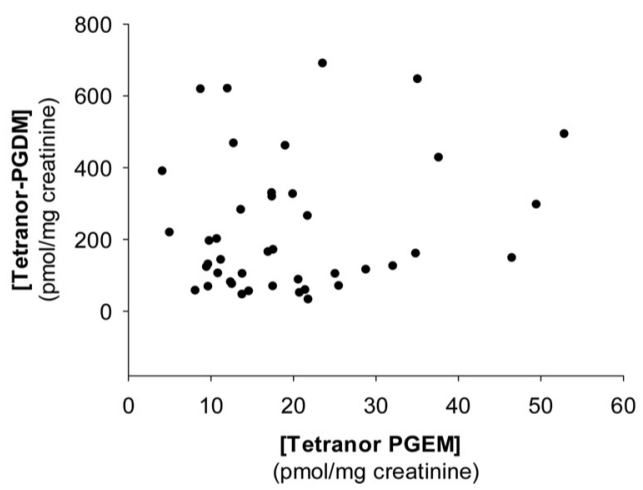

treatment of high-fat diet-induced obesity in mice for 5 days with the selective H-PGDS inhibitor HQL-79 resulted in a significant decrease in food intake. However, this treatment affected neither tissue nor plasma levels of $\mathrm{PGD}_{2}$ and caused only a non-significant trend in the reduction of body weight [12], probably due to a limited treatment period.

The highly increased levels of tetranor-PGDM that we measured here likely reflect an upregulation of the $\mathrm{PGD}_{2}$ pathway in obese human subjects. Whether $\mathrm{PGD}_{2}$ production in humans originates from H-PGDS 


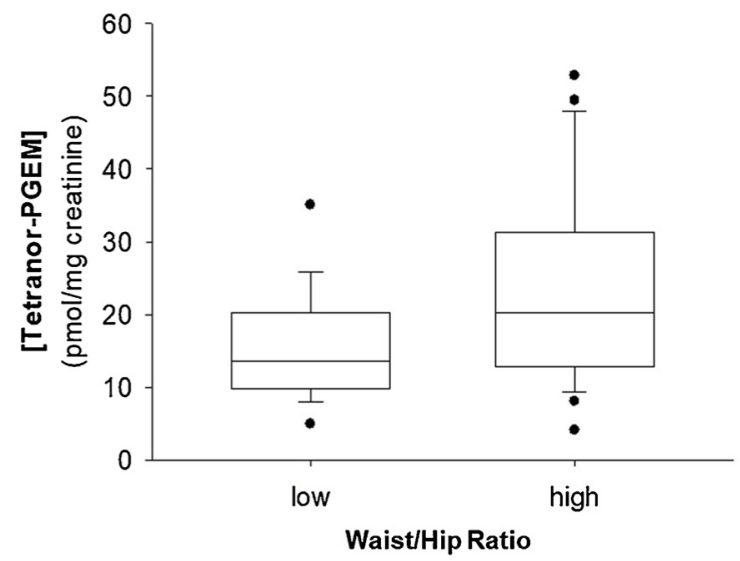

B

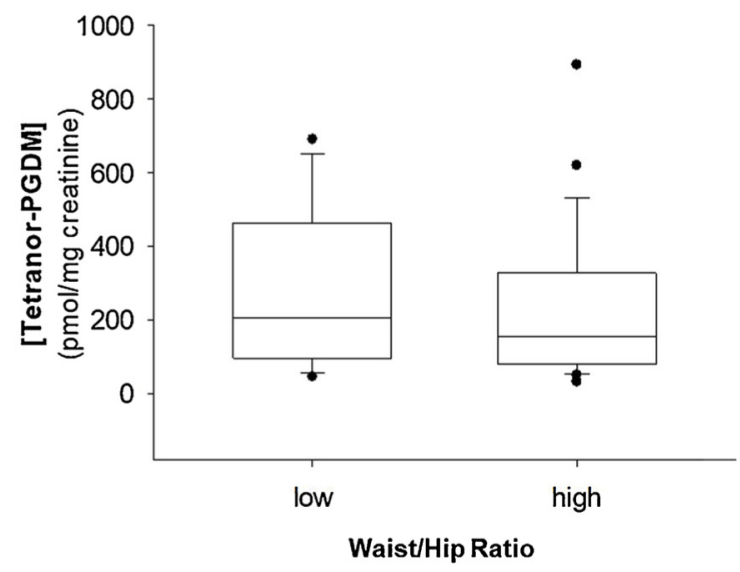

Fig. 2. High levels of urinary tetranor-PGEM correlate with abdominal obesity. Creatinine-normalized urinary prostanoid concentrations in patients stratified by a waist-to-hip ratio (WHR) above or below the WHO-defined threshold for abdominal obesity (low, WHR $<0.90$ for males and $<0.85$ for females; high, WHR $\geq 0.90$ for males and $\geq 0.85$ for females) reveal a significant difference for tetranor-PGEM ( $\mathrm{A}, \mathrm{p}=0.049$ ), but not for tetranor-PGDM ( $\mathrm{B}, \mathrm{p}=0.424$ ).

in the WAT or from L-PGDS in the central nervous system remains to be determined. However, the elevated levels of systemic $\mathrm{PGD}_{2}$ might aggravate obesity in these patients through a possible feedback loop that activates the DP1 receptor and stimulates food intake.

We have determined a rather high ratio of tetranor-PGDM to tetranor-PGEM of $10.6 \pm 3.2$ in this cohort of obese subjects. Other studies that simultaneously measured both of these metabolites reported much higher levels of tetranor-PGEM than tetranor-PGDM in healthy volunteers, resulting in a lower ratio (tetranor-PGDM/tetranorPGEM = 0.13 [31]; 0.33 [32] and Tables 3 and 4). Daham et al. detected that the tetranor-PGDM/tetranor-PGEM ratio is increased approximately 2 -fold in asthmatic patients (0.28 vs. 0.13 ; [31]). Zhang et al. measured the two metabolites in COPD patients and found them to be equally increased, thus not changing the ratio [32]. Therefore, it seems that inflammatory conditions do not generally affect the ratio of the two prostanoids, but increased biosynthesis of $\mathrm{PGD}_{2}$ is rather a specific characteristic of obesity-induced chronic inflammation.

In the context of obesity, the present mass spectrometry-based, targeted lipidomic analysis links the abundance of urinary prostanoids to metabolic parameters in carefully phenotyped obese human subjects. To our knowledge, this has not been investigated before. Despite new insights from this analysis, there are, however, some limitations of this study that need to be acknowledged. In addition to the lack of a control

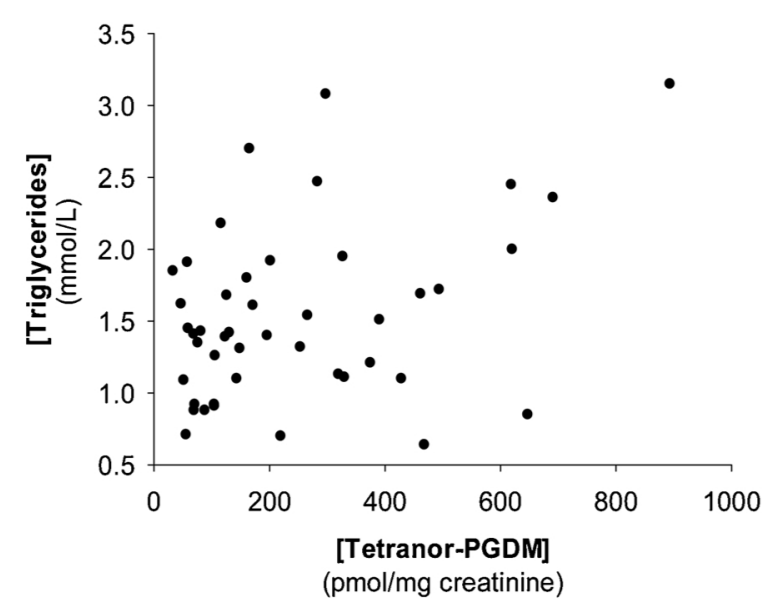

B

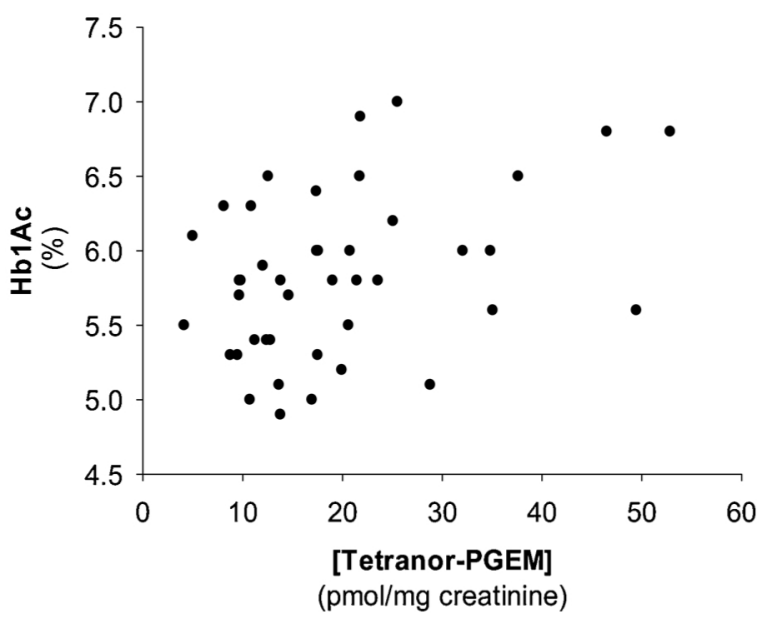

Fig. 3. Urinary prostanoid levels associate with metabolic parameters. (A) Creatinine-normalized tetranor-PGDM concentrations correlate positively and significantly with plasma triglyceride levels $(r=0.381, p=0.0098)$. (B) Creatinine-normalized tetranor-PGEM concentrations correlate positively and significantly with the pre-diabetes marker HbAlc $(r=0.334, \mathrm{p}=0.0286)$.

Table 2

Correlation between tetranor-PGEM and plasma glucose levels.

\begin{tabular}{lll}
\hline & $\mathrm{r}$ & $\mathrm{p}$ \\
\hline Fasting insulin & 0.092 & 0.852 \\
Fasting glucose & 0.211 & 0.211 \\
OGTT T30 & 0.22 & 0.189 \\
OGTT T60 & 0.331 & $\mathbf{0 . 0 4 5}$ \\
OGTT T90 & 0.264 & 0.114 \\
OGTT T120 & 0.168 & 0.285 \\
\hline
\end{tabular}

OGTT, oral glucose tolerance test; T, time (min).

group of non-obese individuals discussed above and a replication cohort, the most obvious limitation is a relatively small sample size of only 45 subjects, which may disguise statistical significance that would be detected in a larger cohort. Furthermore, although systemic evaluation of insulin sensitivity performed by routine measurements is reliable, using state-of-the-art measures, such as hyperglycemic and/or hyperinsulinemic clamp in a larger cohort is warranted in this patient group to confirm the observations for tetranor-PGEM. Finally the 
Table 3

Reported levels of urinary tetranor-PGEM.

\begin{tabular}{|c|c|c|c|c|c|}
\hline control group & $\begin{array}{l}\text { value } \\
\text { (ng/mg creatinine) }\end{array}$ & study group & $\begin{array}{l}\text { value } \\
\text { (ng/mg creatinine) }\end{array}$ & method $^{\mathrm{a}}$ & reference \\
\hline non-obese volunteers before exercise & 2.6 & non-obese volunteers after exercise & 5.3 & LC-MS/MS & [35] \\
\hline healthy volunteers & 7.41 & $\begin{array}{l}\text { healthy smokers or } \\
\text { COPD patients }\end{array}$ & 1.5-2-fold increase & LC-MS/MS & [32] \\
\hline healthy infants & 4.76 & infants with fever & 7.58 & LC-MS/MS & [18] \\
\hline healthy volunteers & 6.74 & asthma patients & 7.9 & LC-MS/MS & [31] \\
\hline
\end{tabular}

${ }^{\text {a }}$ LC-MS/MS, liquid chromatography coupled to tandem mass spectrometry.

Table 4

Reported levels of urinary tetranor-PGDM.

\begin{tabular}{|c|c|c|c|c|c|}
\hline control group & $\begin{array}{l}\text { value } \\
\text { (ng/mg creatinine) }\end{array}$ & study group & $\begin{array}{l}\text { value } \\
\text { (ng/mg creatinine) }\end{array}$ & method $^{\text {a }}$ & reference \\
\hline healthy volunteers & $1.5 \pm 0.3$ & $\mathrm{n} / \mathrm{a}$ & $\mathrm{n} / \mathrm{a}$ & LC-MS/MS & [36] \\
\hline healthy volunteers & 2.47 & $\begin{array}{l}\text { healthy smokers or } \\
\text { COPD patients }\end{array}$ & 1.5-2-fold increase & LC-MS/MS & [32] \\
\hline healthy volunteers & $3.08 \pm 0.15$ & Duchenne muscular dystrophy (DMD) patients & $6.90 \pm 0.35$ & LC-MS/MS & {$[37]$} \\
\hline healthy volunteers & $11.5 \pm 1.7$ & $\begin{array}{l}\text { systemic mastocytosis patients } \\
\text { rheumatoid arthritis patients }\end{array}$ & $\begin{array}{l}37.2 \pm 2.1 \\
20.0 \pm 2.5\end{array}$ & EIA & {$[21]$} \\
\hline healthy volunteers & 0.86 & asthma patients & 2.2 & LC-MS/MS & [31] \\
\hline Aspirin tolerant asthma, baseline & 0.41 & Aspirin tolerant asthma, provocation test & 0.15 & GC-MS & [38] \\
\hline $\begin{array}{l}\text { Aspirin intolerant asthma, baseline } \\
\text { anaphylaxis, baseline }\end{array}$ & $\begin{array}{l}0.75 \\
0.29\end{array}$ & $\begin{array}{l}\text { Aspirin intolerant asthma, provocation test } \\
\text { anaphylaxis, provocation test }\end{array}$ & $\begin{array}{l}\text { 1.9-fold increase } \\
8.5 \text {-fold increase }\end{array}$ & & \\
\hline
\end{tabular}

${ }^{\text {a }}$ LC-MS/MS, liquid chromatography coupled to tandem mass spectrometry; GC-MS, gas chromatography coupled to mass spectrometry; EIA, enzyme immunoassay.

present cohort lacked data on atherosclerosis, which is associated with both metabolic syndrome [33] and an up-regulation of the prostanoid pathway [34]. It can therefore not be excluded that subclinical atherosclerosis may have contributed to the increased urinary prostanoid metabolites in the present study.

In summary, our data disclose an association between urinary prostanoid metabolites and metabolic characteristics of diabetes/prediabetes and lipid metabolism in obese subjects. We performed a targeted lipidomic analysis of urine samples from human subjects without known diabetes and without inflammatory disease that fulfilled criteria of obesity. We correlated the two major prostanoid metabolites that we identified, tetranor-PGEM and tetranor-PGDM, to clinical data. Levels of tetranor-PGDM were markedly elevated in all study subjects and significantly associated with elevated serum triglycerides. TetranorPGEM was associated with abdominal obesity, suggesting that visceral WAT is a dominant source of $\mathrm{PGE}_{2}$ biosynthesis. Furthermore, tetranorPGEM was significantly associated with pre-diabetic measures and impaired glucose tolerance. While these results reassure previous findings from animal studies, they need to be confirmed in larger clinical studies that are able to address and elucidate potential molecular mechanisms involved. In conclusion, the present study bridges some gaps that presently exist between mechanistic in vitro and in vivo studies and clinical data from human subjects.

\section{Contributors}

SPC, AA, AS, and MB conceived the study; SCP and HI performed the mass-spectrometry analysis, $\mathrm{CB}, \mathrm{AS}$, and AA collected clinical data; SCP, AS, and MB drafted the manuscript; PJJ, FSL, and HI critically reviewed the manuscript and provided intellectual input.

\section{Funding}

This work was supported by the Swedish Heart and Lung Foundation (grant number 20180571), King Gustaf V and Queen Victoria Freemason Foundation, Professor Nanna Svartz Foundation, and the Stockholm County Council (grant number 20170365).

\section{Declaration of Competing Interest}

None.

\section{References}

[1] D.W. Haslam, W.P. James, Obesity, Lancet (London, England) 366 (9492) (2005) 1197-1209.

[2] B. Bozkurt, D. Aguilar, A. Deswal, S.B. Dunbar, G.S. Francis, T. Horwich, M. Jessup M. Kosiborod, A.M. Pritchett, K. Ramasubbu, C. Rosendorff, C. Yancy, American Heart Association Heart Failure and Transplantation Committee of the Council on Clinical Cardiology, Council on Cardiovascular Surgery and Anesthesia, Council on Cardiovascular and Stroke Nursing, Council on Hypertension, Council on Quality and Outcomes Research, Contributory risk and management of comorbidities of hypertension, obesity, diabetes mellitus, hyperlipidemia, and metabolic syndrome in chronic heart failure: a scientific statement from the american heart association, Circulation 134 (23) (2016) e535-e578.

[3] S.C. Larsson, A. Wolk, N. Hakansson, M. Bäck, Overall and abdominal obesity and incident aortic valve stenosis: two prospective cohort studies, Eur. Heart J. 38 (28) (2017) 2192-2197.

[4] C.N. Lumeng, S.M. Deyoung, J.L. Bodzin, A.R. Saltiel, Increased inflammatory properties of adipose tissue macrophages recruited during diet-induced obesity, Diabetes 56 (1) (2007) 16-23.

[5] F. Stanke-Labesque, M. Bäck, B. Lefebvre, R. Tamisier, J.P. Baguet, N. Arnol, P. Levy, J.L. Pepin, Increased urinary leukotriene E4 excretion in obstructive sleep apnea: effects of obesity and hypoxia, J. Allergy Clin. Immunol. 124 (2) (2009) 364-370 370 e1-2.

[6] M. Bäck, A. Avignon, F. Stanke-Labesque, C. Boegner, V. Attalin, E. Leprieur, A. Sultan, Leukotriene production is increased in abdominal obesity, PLoS One 9 (12) (2014) e104593.

[7] V. Garcia-Alonso, E. Titos, J. Alcaraz-Quiles, B. Rius, A. Lopategi, C. Lopez-Vicario P.J. Jakobsson, S. Delgado, J. Lozano, J. Claria, Prostaglandin E2 exerts multiple regulatory actions on human obese adipose tissue remodeling, inflammation, adaptive thermogenesis and lipolysis, PLoS One 11 (4) (2016) e0153751.

[8] M. Bäck, L. Yin, E. Ingelsson, Cyclooxygenase-2 inhibitors and cardiovascular risk in a nation-wide cohort study after the withdrawal of rofecoxib, Eur. Heart J. 33 (15) (2012) 1928-1933.

[9] P.J. Jakobsson, S. Thoren, R. Morgenstern, B. Samuelsson, Identification of human prostaglandin E synthase: a microsomal, glutathione-dependent, inducible enzyme constituting a potential novel drug target, Proc. Natl. Acad. Sci. U. S. A. 96 (13) (1999) 7220-7225.

[10] P.O. Hetu, D. Riendeau, Down-regulation of microsomal prostaglandin E2 synthase1 in adipose tissue by high-fat feeding, Obesity (Silver Spring, Md.) 15 (1) (2007) 60-68.

[11] S. Rocha-Rodrigues, A. Rodriguez, I.O. Goncalves, A. Moreira, E. Maciel, S. Santos, M.R. Domingues, G. Fruhbeck, A. Ascensao, J. Magalhaes, Impact of physical exercise on visceral adipose tissue fatty acid profile and inflammation in response to a 
high-fat diet regimen, Int. J. Biochem. Cell Biol. 87 (2017) 114-124.

[12] A. Hernandez-Carretero, N. Weber, M.R. La Frano, W. Ying, J. Lantero Rodriguez D.D. Sears, V. Wallenius, E. Borgeson, J.W. Newman, O. Osborn, Obesity-induced changes in lipid mediators persist after weight loss, Int. J. Obes. (Lond.) 42 (4) (2018) 728-736.

[13] S. Virtue, M. Masoodi, B.A. de Weijer, M. van Eijk, C.Y. Mok, M. Eiden, M. Dale, A. Pirraco, M.J. Serlie, J.L. Griffin, A. Vidal-Puig, Prostaglandin profiling reveals a role for haematopoietic prostaglandin D synthase in adipose tissue macrophage polarisation in mice and humans, Int. J. Obes. (Lond) 39 (7) (2015) 1151-1160.

[14] L. Ragolia, T. Palaia, C.E. Hall, J.K. Maesaka, N. Eguchi, Y. Urade, Accelerated glucose intolerance, nephropathy, and atherosclerosis in prostaglandin D2 synthase knock-out mice, J. Biol. Chem. 280 (33) (2005) 29946-29955.

[15] M. Hamberg, B. Samuelsson, On the metabolism of prostaglandins E 1 and E 2 in man, J. Biol. Chem. 246 (22) (1971) 6713-6721.

[16] E. Granstrom, M. Hamberg, G. Hansson, H. Kindahl, Chemical instability of 15-keto13,14-dihydro-PGE2: the reason for low assay reliability, Prostaglandins 19 (6) (1980) 933-957.

[17] W. Bothwell, M. Verburg, M. Wynalda, E.G. Daniels, F.A. Fitzpatrick, A radioimmunoassay for the unstable pulmonary metabolites of prostaglandin E1 and E2: an indirect index of their in vivo disposition and pharmacokinetics, J. Pharmacol. Exp. Ther. 220 (2) (1982) 229-235.

[18] H. Idborg, S.C. Pawelzik, M. Perez-Manso, L. Bjork, J. Hamrin, E. Herlenius P.J. Jakobsson, Evaluation of urinary prostaglandin E2 metabolite as a biomarker in infants with fever due to viral infection, Prostaglandins, leukotrienes, and essential fatty acids, Prostaglandins Leukot. Essent. Fatty Acids 91 (6) (2014) 269-275.

[19] F. Catella, J. Nowak, G.A. Fitzgerald, Measurement of renal and non-renal eicosanoid synthesis, Am. J. Med. 81 (2B) (1986) 23-29.

[20] L.J. Murphey, M.K. Williams, S.C. Sanchez, L.M. Byrne, I. Csiki, J.A. Oates, D.H. Johnson, J.D. Morrow, Quantification of the major urinary metabolite of PGE2 by a liquid chromatographic/mass spectrometric assay: determination of cyclooxygenase-specific PGE2 synthesis in healthy humans and those with lung cancer, Anal. Biochem. 334 (2) (2004) 266-275.

[21] C. Cho, A. Nguyen, K.J. Bryant, S.G. O'Neill, H.P. McNeil, Prostaglandin D2 metabolites as a biomarker of in vivo mast cell activation in systemic mastocytosis and rheumatoid arthritis, Immun. Inflamm. Dis. 4 (1) (2016) 64-69.

[22] N. Higashi, M. Taniguchi, H. Mita, H. Yamaguchi, E. Ono, K. Akiyama, Aspirin intolerant asthma (AIA) assessment using the urinary biomarkers, leukotriene E4 (LTE4) and prostaglandin D2 (PGD2) metabolites, Allergol. Int. 61 (3) (2012) 393-403.

[23] S. Inagaki, S. Maeda, M. Narita, T. Nakamura, T. Shimosawa, T. Murata, Y. Ohya Urinary PGDM, A prostaglandin D2 metabolite, is a novel biomarker for objectively detecting allergic reactions of food allergy, J. Allergy Clin. Immunol. 142 (5) (2018) 1634-1636 e10.

[24] Y. Arai, S. Arihiro, T. Matsuura, T. Kato, M. Matsuoka, M. Saruta, M. Mitsunaga, M. Matsuura, M. Fujiwara, I. Okayasu, S. Ito, H. Tajiri, Prostaglandin E-major urinary metabolite as a reliable surrogate marker for mucosal inflammation in ulcerative colitis, Inflamm. Bowel Dis. 20 (7) (2014) 1208-1216.

[25] D.R. Matthews, J.P. Hosker, A.S. Rudenski, B.A. Naylor, D.F. Treacher, R.C. Turner,
Homeostasis model assessment: insulin resistance and beta-cell function from fasting plasma glucose and insulin concentrations in man, Diabetologia 28 (7) (1985) 412-419.

[26] G. Klose, F.U. Beil, H. Dieplinger, A. von Eckardstein, B. Foger, I. Gouni-Berthold, F. Heigl, W. Koenig, G.M. Kostner, U. Landmesser, U. Laufs, F. Leistikow, W. Marz, G. Noll, K.G. Parhofer, B. Paulweber, W.F. Riesen, J.R. Schaefer, E. SteinhagenThiessen, A. Steinmetz, H. Toplak, C. Wanner, E. Windler, New AHA and ACC guidelines on the treatment of blood cholesterol to reduce atherosclerotic cardiovascular risk, Wien. Klin. Wochenschr. 126 (5-6) (2014) 169-175.

[27] K.G. Alberti, P.Z. Zimmet, Definition, diagnosis and classification of diabetes mellitus and its complications. Part 1: diagnosis and classification of diabetes mellitus provisional report of a WHO consultation, Diabet. Med. 15 (7) (1998) 539-553.

[28] P.S. Hsieh, J.S. Jin, C.F. Chiang, P.C. Chan, C.H. Chen, K.C. Shih, COX-2-mediated inflammation in fat is crucial for obesity-linked insulin resistance and fatty liver, Obesity (Silver Spring, Md.) 17 (6) (2009) 1150-1157.

[29] K. Ohinata, K. Takagi, K. Biyajima, Y. Fujiwara, S. Fukumoto, N. Eguchi, Y. Urade, A. Asakawa, M. Fujimiya, A. Inui, M. Yoshikawa, Central prostaglandin D(2) stimulates food intake via the neuropeptide Y system in mice, FEBS Lett. 582 (5) (2008) 679-684.

[30] S. Kumar, T. Palaia, C. Hall, L. Ragolia, DP1 receptor agonist, BW245C inhibits dietinduced obesity in ApoE(-/-) mice, Obes. Res. Clin. Pract. 12 (2) (2018) 229-241.

[31] K. Daham, W.L. Song, J.A. Lawson, M. Kupczyk, A. Gulich, S.E. Dahlen, G.A. FitzGerald, B. Dahlen, Effects of celecoxib on major prostaglandins in asthma, Clin. Exp. Allergy 41 (1) (2011) 36-45.

[32] Y. Zhang, G. Zhang, P.A. Clarke, J.T. Huang, E. Takahashi, D. Muirhead, R.C. Steenwyk, Z. Lin, Simultaneous and high-throughput quantitation of urinary tetranor PGDM and tetranor PGEM by online SPE-LC-MS/MS as inflammatory biomarkers, J. Mass Spectrom. 46 (7) (2011) 705-711.

[33] K.B. Won, H.J. Chang, H.C. Kim, K. Jeon, H. Lee, S. Shin, I.J. Cho, S.H. Park, S.H. Lee, Y. Jang, Differential impact of metabolic syndrome on subclinical atherosclerosis according to the presence of diabetes, Cardiovasc. Diabetol. 12 (2013) 41.

[34] V. Capra, M. Bäck, D.J. Angiolillo, M. Cattaneo, K.S. Sakariassen, Impact of vascular thromboxane prostanoid receptor activation on hemostasis, thrombosis, oxidative stress, and inflammation, J. Thromb. Haemost. 12 (2) (2014) 126-137.

[35] M. Blatnik, R.C. Steenwyk, Quantification of urinary PGEm, 6-keto PGF(1alpha) and 2,3-dinor-6-keto PGF(1alpha) by UFLC-MS/MS before and after exercise, Prostaglandins Other Lipid Mediat. 93 (1-2) (2010) 8-13.

[36] W.L. Song, M. Wang, E. Ricciotti, S. Fries, Y. Yu, T. Grosser, M. Reilly, J.A. Lawson, G.A. FitzGerald, Tetranor PGDM, an abundant urinary metabolite reflects biosynthesis of prostaglandin D2 in mice and humans, J. Biol. Chem. 283 (2) (2008) 1179-1188.

[37] T. Nakagawa, A. Takeuchi, R. Kakiuchi, T. Lee, M. Yagi, H. Awano, K. Iijima, Y. Takeshima, Y. Urade, M. Matsuo, A prostaglandin D2 metabolite is elevated in the urine of Duchenne muscular dystrophy patients and increases further from 8 years old, Clin. Chim. Acta 423 (2013) 10-14.

[38] N. Higashi, H. Mita, H. Yamaguchi, Y. Fukutomi, K. Akiyama, M. Taniguchi, Urinary tetranor-PGDM concentrations in aspirin-intolerant asthma and anaphylaxis, J. Allergy Clin. Immunol. 129 (2) (2012) 557-9, 559 e1-2. 